\title{
The Cancer Stem Cell Marker GSK3b, a Gene involved in Oxidative Stress, Modulates Chemoresistance in Ovarian Cancer
}

\author{
Noelle Cutter \\ Julia Fiederlein \\ Brianna McNulty \\ Cassandra Greco \\ Kimberly Doyle \\ Molloy College \\ 1000 Hempstead Avenue, Rockville Centre, NY \\ United States of America
}

\begin{abstract}
The leading cause of death from gynecologic malignancies is epithelial ovarian cancer. These tumors are comprised of a highly heterogeneous population of cells, of which only a small subset of stem-like cells possess the ability to regenerate tumors in vivo. These cancer stem cells (CSCs) represent a significant clinical challenge as they are resistant to conventional cancer therapies and play essential roles in metastasis and tumor relapse. While chemotherapy is the preferred treatment modality, chemoresistance severely limits treatment success. It has been hypothesized that cancer stem cells are at the root of this problem. Their ability to self-renew and proliferate is what causes a large number of ovarian cancers to recur and not respond to normal chemotherapeutic treatments. Recent evidence suggests that deregulation of stem cell pathways is a key factor in the onset and maintenance of chemoresistance. Several key markers such as BMI1, FZD1, GGSK3b, NANOG, TWIST1, and OCT4 (POU5F1) are hypothesized to play a central role in the development and differentiation of multiple cell lineages. Recent studies have demonstrated that these markers is required for the carcinogenesis in several cancer types. The aim of this study was to investigate the significance of CSC expression in chemoresistant and sensitive ovarian cancer cancer cell lines. Through the analysis of gene expression in these cell lines, CSC markers could be identified. CSC mRNA expression was detected by real-time quantitative PCR and by gel electrophoresis of PCR products in sensitive and resistant ovarian cell lines. The objective of such an improved delineation is to develop targeted therapy for selective elimination of cancer stem cells with minimal toxicity to normal stem cells. Our research indicates that there were novel changes in gene expression in the chemoresistant cell lines, specifically involving GSK3b. Further research is needed to gain better understanding of the role that these genes specifically play in cancer survival, metastasis, and chemo resistance.
\end{abstract}

Keywords: biology, ovarian cancer, cancer stem cells, epigenetics, cancer stem cell markers, chemoresistance

\section{Introduction:}

Epithelial ovarian cancer is the result of the ovarian surface epithelium cells becoming malignant (Hennessy 2009). Every year 220,000 women develop epithelial ovarian cancer worldwide. In the United States, it is considered to be the gynecologic malignancy with the most fatalities (Seiden 2001). Pelvic pain, bloating, and frequent urination are among the most common symptoms of the disease (Walker 2018). The symptoms are quite nonspecific, which causes numerous cases to remain undiagnosed (Sun 2016). There are four main stages of this disease, and various substages. In Stage IA, the tumor is limited to one ovary. The tumor is located in both ovaries in Stage IB. Stage IC is diagnosed when the tumor is limited to one or two ovaries with signs such as capsule rupture, tumor on ovarian surface, and/or malignant cells in ascites. Stage II of epithelial ovarian cancer is characterized by the spreading of the tumor to the pelvis. The tumor spreads to the uterus and fallopian tubes in Stage IIA, and extends to the other pelvic tissues in Stage IIB (Jayson 2014). Ovarian cancer tumors that are diagnosed between stages I and IIA generally have the most promising survival rates, with approximately $80 \%$ of women living 5 years or more post-diagnosis (Burges 2011). Pelvic extension with malignant cells in ascites occurs during Stage IIC. Stage III of ovarian cancer involves presence of the tumor in one or both ovaries and shows microscopically confirmed peritoneal implants outside of the pelvis leading to abdominal disease. 
Stage IIIA shows microscopic metastases beyond the pelvis, while Stage IIIB shows macroscopic peritoneal metastases beyond the pelvis with $2 \mathrm{~cm}$ or less. Peritoneal metastases beyond the pelvis with $2 \mathrm{~cm}$ or greater are evident in Stage IIIC. Finally, Stage IV tumors show signs of distant metastases to the liver. (Jayson 2014). At this late stage, less than $10 \%$ of patients live 5 years or more after diagnosis (Huang 2017).

Most of the women diagnosed with this disease are found to have stage III (Jayson 2014). In these cases, treatment usually involves tumor debulking surgery, followed by a platinum-based chemotherapy regimen (Cortez 2017). While treatment is often very successful, ovarian cancer still has a very high death rate. This is due to the late presentation of symptoms. It is also due in part to the fact that women that are diagnosed with the advance disease or stage III will often develop episodes of recurrent disease with a shorter disease-free interval, typically within 18 months of treatment. (Jayson, 2014). The survival rate for women diagnosed with the advance disease is only 25\% compared to those with the early stage whose survival rate is typically $80-90 \%$. Outcomes can be improved if the tumor is detected at an early stage of development. Unfortunately, this disease does not produce any ovarian cancer-specific symptoms in the early stages, which is why it is difficult to diagnose the disease early on (Colombo 2006). Recurrence arises when cell populations evade the effects of chemotherapy. Non-proliferative, or quiescent, cancer cells are resistant to paclitaxel and carboplatin. Although a general problem for all ovarian cancers, recurrence is variable among subtypes. The majority (90\%) of ovarian cancers develop in the epithelium with the highest rate of recurrence.

A subpopulation of tumor cells that have the capacity to differentiate, self-renew, and produce a tumor upon implantation into an animal host are known as cancer stem cells (Yu 2012). Cancer stem cells are also commonly referred to as tumor initiating cells (Parte 2018). It is hypothesized that cancer stem cells arise from mutated stem cells, although studies have shown that mutated progenitor cells might be responsible (Jordan 2006). Recurrence of ovarian cancer tumors post-treatment could be due to the presence of these cancer stem cell populations, as they are generally chemoresistant (Beck 2013). These cells acquire resistance through a variety of mechanisms. Many platinum based drugs induce tumor regression by causing DNA damage to the cancer cell. Cancer cells often have defective DNA repair pathways. The rapid proliferation of these cells is often in S-phase, which is a vulnerable phase for DNA damage. Due to the chemotherapy drugs, these cells are not able to repair from the DNA damage. However, studies show that cancer stem cells have elevated levels of DNA repair. This provides an explanation as to potentially why they become resistant. (Thomas 2014). Additionally, cancer stem cells have a slow proliferation rate. Because chemotherapy drugs are designed to target rapidly dividing cells, the cancer stem cell population is often unaffected (Nunes 2018). Because cancer stem cells prevent chemotherapy from completely eradicating tumors, various research projects have focused on determining how cancer stem cells function on the epigenetic level. Specifically, the identification of cancer stem cell markers could allow for cancer stem cell identification and improved ovarian cancer diagnostics (Paula 2017). Previous studies have determined that CD133, CD44, CD117, and CD24 are ovarian cancer stem cell markers (BurgosOjeda 2012), however, many other ovarian cancer stem cell markers that could prove to be useful in the diagnosis and treatment of the disease remain unidentified.

A consideration of the unique cellular and environmental features of chemoresistant ovarian cancer cells could shed light on potential therapeutic improvements targets. The aim of this study was to analyze the gene expression changes in chemoresistant and chemosensitive human cell lines of several cancer stem cell markers thought to play an important role in ovarian cancer chemoresistance.

Our study revealed several gene expression changes, primarily involving the stem cell marker GSK3b. GSK3b plays a role in apoptotic mechanisms and the regulation of oxidative stress. The inhibition of GSK3b expression is crucial in maintaining the stability of Nrf2. Nrf2 prevents oxidative stress, which is a necessary measure in the maintenance of healthy, undamaged cellular DNA. Over expression of GSK3b leads to uncontrolled oxidative stress, and therefore, cancer stem cell proliferation (Mancinelli 2017). We hypothesize that understanding the molecular mechanisms underlying this drug resistance is important for the development of effective therapies to improve ovarian cancer patients' outcome.

\section{Materials and Methods:}

\subsection{Materials}

HOSE 6-3, a normal human ovarian surface epithelial cell line that has been immortalized by HPV E6/7 ORF (obtained from Dr. S. W. Tsao, Cold Spring Harbor Laboratory, NY) cell line was used to study ovarian cancer proliferation. All cell lines were maintained in MEM 10\% fetal bovine serum. SYBR Green I fluorescent dye, reverse transcriptase and RNase inhibitor was purchased from BiMake. BioRad supplied the cDNA conversion kit.

RNEasy, DNEasy obtained from Qiagen. Cisplatin and Carboplatin were purchased from Sigma. Primers were supplied by Sigma Genosys. Cell counting kit- 8 was purchased from Dojindo Molecular Technologies, Inc. 


\subsection{Cell lines}

Several human cell lines were used in this study. SKOV3 and CA-CRSPR were the chemoresistant cell lines, CAOV3 was the chemosensitive cell line, and HOSE was the control. SKOV3 and CAOV3 are human ovarian cancer cell lines derived from ovarian epithelium. All cell lines were maintained in minimum essential medium- $\alpha$ supplemented with $10 \%$ FBS (Invitrogen) in the presence of $100 \mathrm{U} / \mathrm{mL}$ of penicillin and $100 \mu \mathrm{g} / \mathrm{mL}$ of streptomycin, and were incubated at $37^{\circ} \mathrm{C}$ with 5\% CO2. The cultured cells were split every 2 to 3 days and maintained in an exponential growth phase. All cell lines were purchased at ATCC. These cell lines have been routinely tested for Mycoplasma contamination in the laboratory.

\subsection{Pathway Analysis}

DAVID, Ingenuity, gPROFILER are bioinformatics database is used to analyze large gene/protein lists and used to study in order to gain biological meaning. Analysis was useful in gaining a better understanding of candidate genes involved in chemoresistance in ovarian cancer. Once an uploaded gene list is obtained, analysis can be performed in order to classify the genes based on different mechanisms and pathways that are good for our study. PubMed metaanalysis can be done with smaller gene lists of interest to gain information on chemoresistant mechanisms.

\subsection{Cell Counting Kit-8}

Cell Counting Kit-8 (CCK-8) is a colorimetric assays for the determination of cell viability in cell proliferation and cytotoxicity assays. The water-soluble tetrazolium salt, WST-8, is reduced by dehydrogenase activities in cells to give a yellow-color formazan dye, which is soluble in the tissue culture media. The amount of the formazan dye, generated by the activities of dehydrogenases in cells, is directly proportional to the number of living cells. We used it to measure cell proliferation where increased absorbance using a spectrophotometer correlates to increased cell death. The BioTek 800 plate reader was used to measure absorbance at $450 \mathrm{~nm}$ filter.

\subsection{EarlyTox Live/Dead Assay}

This live/dead assay kit utilizes two cell markers that fluoresce based on the integrity of a cells membrane. Calcein AM was the marker used for live cells and ETHD-III for dead cells. Calcein AM permeates a live, intact cell membrane and is there converted into its green fluorescent form, Calcein. The population of live cells was calculated based on the intensity of this green fluorescence (emission 495-530 nm). ETHD-III was used as a dead-cell marker because is impermeable to intact cell membranes and enters broken or compromised cell membranes where it binds to nucleic acids and fluoresces red. The population of dead cells was calculated based on the intensity of this red fluorescence (emission 530-645 nm). Fluorescence was measured using the SpectraMax microplate reader.

\subsection{Primers}

A variety of different primers were utilized in this study to test their expression in the aforementioned cell lines. Each primer was used as a component of the qPCR master mix. The master mix contained a forward and reverse sequence of each primer. The complete list of primers used in this experiment can be found in Figure 1.

\subsection{DNA Extraction}

DNA extraction is the process by which DNA is separated from proteins, membranes, and other cellular material contained in the cell from which it is recovered (Elkins 2013).

We began our DNA extraction by pipetting 20 ul QIAGEN protease into a 1.5 microcentrifuge tube. $200 \mathrm{ul}$ of the sample and another $200 \mathrm{ul}$ of the buffer were added to the tube and mixed by vortexing for 15 seconds. The tube was incubated at 56C for 10 minutes. $200 \mathrm{ul}$ of ethanol were added next and mixed by vortexing another 15 seconds. Next it was centrifuged for a minute and the tube containing the filtrate was discarded. Buffer was added next and then centrifuged for another minute. The previous step was repeated three times until the tube was incubated at room temperature for a minute then centrifuged one last time at $8000 \mathrm{rpm}$. Incubating at room temperature before centrifugation generally increases DNA yield.

\subsection{PCR}

Polymerase Chain Reaction (PCR) uses DNA polymerase to amplify a small DNA sample by synthesizing multiple new copies (Garibyan 2013). DNA polymerase requires a primer to allow for the addition of the first nucleotide onto a 3'-OH group. After the PCR reaction is completed, the DNA sample is amplified into billions of copies ("Polymerase Chain Reaction" 2017). PCR products can be visualized through gel electrophoresis. Following DNA extraction, SKOV3 and HOSE DNA were run through a PCR machine in order to amplify the sequences. Prior to PCR, the DNA samples were mixed with certain primers. A PCR Master Mix was made for a total of 12 reactions. Two master mix solutions were made up, one for each DNA type. The master mix contained SKOV3 or HOSE DNA, Master Mix solution and distilled water. 
The DNA was inserted into PCR tubes. Each tube within the strip contained a forward primer and a reverse primer. The primers used were ACT, SOX9, FDZ1, POU5F1 (OCT4), NOTCH3, SLUG, GSK3b and ZEB1. The PCR tubes were ran in the PCR machine for DNA amplification. Finally, the PCR tubes were ran on a gel electrophoresis for visualization. The PCR was performed following manufacturer's protocol.

ACT, forward, 5'-TTGCAGCTCCTTCGTTGC-3'; reverse, 5'-GTGGTACGACCAGAGGCATAC-3'; SOX9, forward, 5'-GACGCTGGGCAAGCTCT-3'; reverse, 5'-GTAATCCGGGTGGTCCTTCT-3'; FDZ1, forward, 5'CAGCAGTACAACGGCGAAC-3'; reverse, 5'-GTCCTCCTGATTCGTGTGGC-3'; POU5F1 (OCT4), forward, 5'AGTTGGCGTGGAGACTTTGC-3'; reverse, 5'-CAGGGCTTTCATGTCCTGG-3'; NOTCH3, forward, 5'GTGTGTGTCAATGGCTGGAC-3'; reverse, 5'-GTGACACAGGAGGCCAGTCT-3'; SLUG， forward, 5'CTGCGGCAAGGCGTTT-3'; reverse, '5-CGTGTGAGTTCTAATGTGTCCTTGA-3'; GSK3b, forward, 5'GGAACTCCAACAAGGGAGCA-3'; reverse, 5'-TTCGGGGTCGGAAGACCTTA-3'; ZEB1, forward, 5'TTCACAGTGGAGAGAAGCCA-3'; reverse, 5'-GCCTGGTGATGCTGAAAGAG-3'.

\subsection{Gel Electrophoresis}

Gel electrophoresis is a technique in nucleic acid biochemistry that involves the separation of molecules based on their size (Chadalavada 2009). During this experiment, $1 \%$ agarose gels with ethidium bromide were used to separate DNA in a 1x tris-acetate buffer. $10 \mu 1$ of the DNA (SKOV3 or HOSE) from the PCR tubes were mixed with $5 \mu 1$ of blue dye. A total of $15 \mu \mathrm{l}$ was loaded into each well. Samples were run at $100 \mathrm{~V}$ for approximately 45 minutes and visualized using a UV-transilluminator with a camera.

\subsection{0 cRNA Extraction}

RNA extraction was performed following the IBI Scientific protocol. In short, first cell lysis was done to break up the tissue pellet. The mixture was transferred to a collection tube with a filter column before being centrifuged at a speed of $1,000 \mathrm{x} \mathrm{g}$. RNA binding was step two of the process, involving the addition of $70 \%$ ethanol and centrifugation at $16,000 \times \mathrm{g}$ using a new collection tube. The flow-through in the collection tube was discarded. Step three, wash, used the Wash Buffer, followed by several rounds of centrifugation at a speed of 14-16,000 x g. The flow-through was discarded following each wash and centrifugation. The process ends with step four, RNA elution. RNase-free water was added to the center of the filter tube and allowed to sit and absorb for approximately two minutes before being centrifuged. This final centrifugation left behind the purified RNA that would be converted to cDNA to be used for qPCR.

\subsection{RNA to cDNA Conversion}

In order for a qPCR to be performed, RNA must be converted to cDNA. Three microliters of template RNA, two microliters of mixed primers, four microliters of master buffer, and 11 microliters of distilled water were combined and put into the PCR machine so that the RNA would be converted to cDNA. The exact protocol used can be found in Figure 2.

\section{$2.12 \mathrm{qPCR}$}

The qPCR, or quantitative polymerase chain reaction, is commonly used in laboratories to quantify gene expression in a particular sample (Adamski 2014). Each qPCR required the use of a master mix. The master mix contained SYBR green, ROX reference, distilled water, and the forward and reverse primer of each gene. The master mix was created according to the Bimake ${ }^{\mathrm{TM}}$ 2x SYBR Green qPCR Master Mix protocol.

The same amounts of each component were used for each trial, as the concentrations of all of the cDNA samples used were extremely similar. Because the samples were to be plated in triplicate, the master mix protocol was tripled. The mixture was plated on a 96 well plate. To quantify and detect nucleic acids in the SKOV3 and CAOV3 cell lines. Five trials of qPCR were performed in accordance with the Bimake ${ }^{\mathrm{TM}} 2 \mathrm{x}$ SYBR Green qPCR Master Mix protocol. Total cellular RNA was isolated using RNeasy kit and 1ug of RNA was reverse transcribed to cDNA using the SuperScript One Step RT-PCR system by Invitrogen, according to the manufacturer's instructions. cDNA at a 1:10 dilution was used for all PCR reactions and primers were designed by Sigma (see primer sequences above). Each qPCR was performed in triplicate to obtain the most accurate measurements possible, as errors could be made during the pipetting process.

After the 96 well plate was loaded with the master mix and inserted into the qPCR machine, the temperature settings were set based on the Bimake ${ }^{\mathrm{TM}}$ protocol. These temperature settings, along with the steps they coincide with, can be found in Figure 3.1. All PCR reactions were performed on CFX-96 Bio-Rad RT System in and validated by the presence of a single peak in the melt curve analysis. Changes in gene expression were calculated relative to the actin and/or HPRT control. 


\section{Results:}

\subsection{Cell Counting Kit-8}

CCK- 8 assays were performed using a 48 -well plate. It was performed in triplicate in order to obtain accurate data. The values for each treatment type obtained from the machine were averaged together to determine a mean cell viability value. Cell viability was found to be highest in dual treated cells (mean: 0.42), and lowest in the carboplatin treated cells (mean: 0.13). Figure XX illustrates the cell viability values for non-treated, carboplatin treated, cisplatin treated, and dual treated SKOV3 cells.

\subsection{Early Tox Live/Dead Analysis}

To determine sensitivity of the cell lines to the various chemotherpay reagents, the EarlyTox live/dead assay was used. As you can see from the results, treatment with either cisplatin (CIS+), carboplatin (CPT+), or dual (CIS+ and CPT+) resulted in chemoresistance of SKOV3 as compared to the chemosensitive HOSE control population. CAOV3 exhibited a medial sensitivity.

\subsection{Gel electrophoresis}

After running the Gel electrophoresis on the two different DNA cell lines, SKOV3 and HOSE DNA, it was determined that some of the primers were expressed in a particular cell line, while others were not. The ACT and SOX9 primers were not expressed in either the chemoresistant cell line, SKOV3, or the chemosensitive cell line, HOSE. The FZD1 primer was expressed in both cell lines. POU5F1, NOTCH3, SLUG, GSK3b and ZEB1 were expressed in the chemoresistant cell line but not in the sensitive cell line. Figure 4 outlines the expression levels of each primer tested in SKOV3 and HOSE based upon the results of the gel electrophoresis experiments.

\section{$3.4 \mathrm{qPCR}$}

After performing five qPCR trials, several cq values were obtained for each gene. These values were averaged together to get a general representative $\mathrm{cq}$ value. A cq value above 32 indicates underexpression of the target gene, while a cq value of below 28 indicates overexpression of the target gene. The results were graphed for facilitated visualization of the data. Some cq values were not graphed, such as in the case of KLF4, as the results were inconclusive with values ofboth over and underexpression. See Figure 7 and 8.

\section{Discussion}

Chemoresistance is a major cause of tumor recurrence and can potentially be attributed to cancer stem cells. The CCK8 assay performed in this study was used to determine the levels of SKOV3 cell viability and proliferation following platinum-based chemotherapy treatment. Carboplatin and cisplatin are two major platinum-based chemotherapy drugs used in the clinical setting. Based on the data collected from running the CCK-8 assay, it was found that carboplatin is the most effective chemotherapeutic drug for treatment of chemoresistant cells of the SKOV3 cell line. The SKOV3 cells treated with carboplatin displayed the lowest levels of viability and proliferative ability. Cisplatin did not cause a significant difference in cell viability and proliferation, as compared to the non-treated SKOV3 cells. While further research is necessary to determine the most effective treatment, our study found that carboplatin had the greatest impact on cell viability and proliferation of chemoresistant SKOV3 cells. This data could potentially be useful in the clinical setting when treating ovarian cancer. The primary aim of this study was to identify the genes that are potentially playing a role in the chemo resistance of ovarian cancer stem cells. These genes could be considered to be cancer stem cell markers. The gel electrophoresis and PCR experiments performed following DNA extraction allowed for the analysis of cancer stem cell gene expression. The extracted DNA from HOSE (Human Ovarian Surface Epithelial) DNA and SKOV3 DNA were mixed with primers and then ran through a PCR machine for copying. Following the gel electrophoresis, some of the primers were found to be expressed in the DNA while others were not. There are many different reasons behind the minimal to no expression of the DNA in the gel, such that the primer/DNA is weak or the PCR ran at a wrong frequency. HOSE cell line was the control cell line. SKOV3 cell line is the chemoresistant as it resists the action of a chemical agent, however HOSE and A2780 are chemosensitive and are receptive to change to the action of a chemical agent. Primers such as POU5F1, NOTCH3, SLUG, GSK3b, and ZEB1 were found to have different expression levels in the chemoresistant SKOV3 cell line, as compared to the control HOSE cell line. These results indicate that POU5F1, NOTCH3, SLUG, GSK3b, and ZEB1 expression was altered in chemoresistant ovarian cancer stem cells and could therefore potentially be used as diagnostic markers. There is significance to the altered expression of these specific genes in the chemoresistant SKOV3 cell line, as compared to HOSE. POU5F1 has previously been found to be expressed in tumor initiating cells. 
Tumor initiating cells are responsible for tumor initiation, chemoresistance, and tumor metastasis (Wang 2015). In this study, POU5F1 was found to be expressed only in the chemoresistant cell line. POU5F1 expression in chemoresistant ovarian cancer cells could modulate the tumor initiation, metastasis, and lack of chemotherapeutic response. NOTCH 3 is widely known to play a role in apoptosis. Apoptosis, or programmed cell death, occurs as a result of DNA damage, or when something goes wrong during the cell cycle. It is the body's way of ensuring that damaged cells will not replicate. When apoptosis does not occur, damaged cells can replicate and cancer often results. Because NOTCH3 plays a role in apoptosis, altered NOTCH3 expression can lead to suppression of apoptosis, and therefore, cancer. NOTCH3 expression was altered in SKOV3 cells, indicating that this gene is potentially allowing for tumor initiation. SLUG, another gene with altered expression in SKOV3, has previously been linked to tumor progression (Ganesan 2016). Altered expression of a gene involved in tumor progression, such as SLUG, can potentially explain why cancer stem cells metastasize effortlessly. GSK3b was found to also have altered expression in SKOV3. This gene in particular plays various roles when considering cancer genetics. Some studies have found altered GSK3b expression is involved in cancer progression and inhibition of apoptotic processes. Additionally, it is involved in oxidative stress management within the cell. The inhibition of GSK3b helps maintain the stability of Nrf2, a transcription factor necessary for defense against oxidative stress. Overexpression of GSK3b, therefore, leads to cellular oxidative stress (Mancinelli 2017). Oxidative stress within a cell is associated with increased DNA damage, mutations, cancer cell metastasis, and overall genomic instability (Reuter 2010). The results of this study indicate that GSK3b is likely involved in cancer progression due to oxidative stress and apoptosis inhibition in ovarian cancer, as it was expressed in only the chemoresistant cell line. ZEB1 is known in the scientific community to play a role in tumorigenicity. Studies have shown that it is also linked to chemoresistance in breast cancer (Zhang 2018). Breast cancer is closely linked to ovarian cancer when considering genetics. Being that ZEB1 has been found to be linked to chemoresistance in breast cancer, it is likely that is also has this same role in ovarian cancer, especially when considering its altered expression in the chemoresistant ovarian cancer cell line. It is evident that these genes plays a role in the progression of cancer, and their altered expression in SKOV3 cells indicates that they are likely markers of chemoresistant ovarian cancer stem cells.

Based on analysis of the data obtained from running five trials of qPCR, it was determined that three genes might have a link to the chemoresistance of these cancer stem cells. The altered expression of GSK3b, CD34 and FZD1in the resistant SKOV3 cell line lead to further analysis of the functions of these genes in order to determine why this might be. As previously stated, overexpression of GSK3b leads to increased oxidative stress. According to the qPCR data, this gene had altered expression in the chemoresistant SKOV3 cell line, as compared to the chemosensitive CAOV3 cell line. A gene such as GSK3b being overexpressed in a chemoresistant cell line could explain why the chemoresistant ovarian cancer cells have an increased ability to proliferate, as the oxidative stress likely induced DNA mutations. CD34 has previously been found to be overexpressed in response to cellular proliferation. Therefore, it has been considered to be a marker of activated stem cells (Goodell 1999). Our qPCR data indicated that CD34 was, in fact, overexpressed in the chemoresistant cell line, as compared to the chemosensitive cell line. It was expected for CD34 to be overexpressed in the SKOV3 cell line, as cancer stem cells are proliferative cells. This data allows for further confirmation of the status of CD34 as a cancer stem cell marker in ovarian cancer. FZD1 plays a crucial role in multidrug chemoresistance. The Wnt/beta-catenin pathway regulates tumor proliferation, and FZD1 is a protein involved in this pathway. Studies have shown that when FZD1 is overexpressed in breast cancer, multidrug chemoresistance is the result (Zhang 2012). Breast cancer and ovarian cancer are closely linked genetically. Our data indicated that FZD1 expression was altered in chemoresistant SKOV3 cells, as compared to chemosensitive CAOV3 cells. The altered expressed of FZD1 in the SKOV3 cell line could be responsible for its chemoresistant abilities.

The aforementioned genes can potentially be used as markers for diagnosis of ovarian cancer. Specifically, GSK3b is a potential candidate for an ovarian cancer stem cell diagnostic marker, as its altered expression remained consistent in both the gel electrophoresis and qPCR experiments. While the data collected from this study looks promising, more trials need to be performed to confirm that this gene can be used as a diagnostic marker.

Additionally, more qPCR trials should also be performed using the same primers and cDNA samples to obtain more accurate results. There is hope that with future research, the information gathered on this gene and its expression in chemoresistant cell lines through this study can be brought into the clinical setting to diagnose and treat ovarian cancer.

\section{References}

Adamski, Mateusz G., Patryk Gumann, and Alison E. Baird. "A Method for Quantitative Analysis of Standard and High-Throughput QPCR Expression Data Based on Input Sample Quantity." PLoS ONE 9, no. 8 (2014). Accessed March 31, 2019. doi:10.1371/journal.pone.0103917. 
Beck, B., \& Blanpain, C. (2013). Unravelling cancer stem cell potential. Nature Reviews.Cancer, 13(10), 727-38. doi:http://dx.doi.org.molloy.idm.oclc.org/10.1038/nrc3597

Burges, Alexander, and Barbara Schmalfeldt. "Ovarian Cancer." Deutsches Aerzteblatt Online, 2011. Accessed March 31, 2019. doi:10.3238/arztebl.2011.0635.

Burgos-Ojeda, Daniela, Bo R. Rueda, and Ronald J. Buckanovich. "Ovarian Cancer Stem Cell Markers: Prognostic and Therapeutic Implications." Cancer Letters 322, no. 1 (2012): 1-7. Accessed March 31, 2019. doi:10.1016/j.canlet.2012.02.002.

Chadalavada, Durga M., and Philip C. Bevilacqua. "Analyzing RNA and DNA Folding Using Temperature Gradient Gel Electrophoresis (TGGE) with Application to In Vitro Selections."

Methods in Enzymology Biophysical, Chemical, and Functional Probes of RNA Structure, Interactions and Folding: Part A, 2009, 389-408. doi:10.1016/s0076-6879(09)68018-6.

Colombo N, Van Gorp T, Parma G, Amant F, Gatta G, Sessa C, Vergote I. 2006. Ovarian cancer. Critical Reviews in Oncology/Hematology. 60(2):159-79.

Cortez, Alexander J., Patrycja Tudrej, Katarzyna A. Kujawa, and Katarzyna M. Lisowska. "Advances in Ovarian Cancer Therapy." Cancer Chemotherapy and Pharmacology 81, no. 1 (2017): 17-38. Accessed March 31, 2019. doi:10.1007/s00280-017-3501-8.

Donaldson, C. D., and K. N. Bishop. "Cell Culture." British Journal of Hospital Medicine 76 (2015): C2-5.

Garibyan, Lilit, and Nidhi Avashia. "Polymerase Chain Reaction." Journal of Investigative Dermatology 133, no. 3 (2013): 1-4. doi:10.1038/jid.2013.1.

Ganesan, Ramya, Elizabeth Mallets, and Julian Gomez-Cambronero. "The Transcription Factors Slug (SNAI2) and Snail (SNAI1) Regulate Phospholipase D (PLD) Promoter in opposite Ways towards Cancer Cell Invasion." Molecular Oncology 10, no. 5 (2015): 663-76. doi:10.1016/j.molonc.2015.12.006.

Goodell, Margaret A.. "CD34+ or CD34-: Does it Really Matter?." Blood 94, no. 8 (1999): 2545-2547. Accessed June 06, 2019.http://www.bloodjournal.org/content/94/8/2545.

Hennessy, Bryan T., Robert L. Coleman, and Maurie Markman. "Ovarian Cancer." The Lancet 374, no. 9698 (September 28, 2009): 1371-382. Accessed March 15, 2019. doi:https://doi.org/10.1016/S01406736(09)61338-6.

Huang, Zhen, Hua Yan, Devendra Chavan, Zeng Yuan, Xingsheng Yang, Youzhong Zhang, Kun Song, and Beihua Kong. "Effective Treatment of a Patient with Stage IV Ovarian Cancer: A Case Report." Oncology Letters, 2017. Accessed March 31, 2019. doi:10.3892/ol.2017.7285.

Jayson GC, Kohn EC, Kitchener HC, Ledermann JA. 2014. Ovarian cancer. The Lancet. 384(9951):1376-88.

Jordan, C. T., PhD., Guzman, M. L., PhD., \& Noble, M., PhD. (2006). Cancer stem cells. The New England Journal of Medicine, 355(12), 1253-61. Retrieved from http://molloy.idm.oclc.org/login?url=https://search-proquestcom.molloy.idm.oclc.org/docview/223932639?accountid=28076

Mancinelli, Romina, Guido Carpino, Simonetta Petrungaro, Caterina Loredana Mammola, Luana Tomaipitinca, Antonio Filippini, Antonio Facchiano, Elio Ziparo, and Claudia Giampietri. "Multifaceted Roles of GSK-3 in Cancer and Autophagy-Related Diseases." Oxidative Medicine and Cellular Longevity 2017 (2017): 1-14. doi:10.1155/2017/4629495.

Margaret Lois Thomas, Krysta Mila Coyle1, Mohammad Sultan1, Ahmad Vaghar-Kashani1 and Paola Marcato2*. 2014. Chemoresistance in cancer stem cells and strategies to overcome resistance. Chemotherapy: Open Access. 3(1).

Nunes, Toni, Diaddin Hamdan, Christophe Leboeuf, Morad El Bouchtaoui, Guillaume Gapihan, Thi Nguyen, Solveig Meles, Eurydice Angeli, Philippe Ratajczak, He Lu, Mélanie Di Benedetto, Guilhem Bousquet, and Anne Janin. "Targeting Cancer Stem Cells to Overcome Chemoresistance." International Journal of Molecular Sciences 19, no. 12 (2018): 4036. Accessed March 31, 2019. doi:10.3390/ijms19124036.

Parte, Seema C., Surinder K. Batra, and Sham S. Kakar. "Characterization of Stem Cell and Cancer Stem Cell Populations in Ovary and Ovarian Tumors." Journal of Ovarian Research 11, no. 1 (2018). Accessed March 31, 2019. doi:10.1186/s13048-018-0439-3.

Paula, Arnaud Da Cruz, and Carlos Lopes. "Implications of Different Cancer Stem Cell Phenotypes in Breast Cancer." Anticancer Research 37, no. 5 (2017): 2173-183. doi:10.21873/anticanres.11552.

Polymerase chain reaction (PCR). Ncbi. (2017).

Reuter, Simone, Subash C. Gupta, Madan M. Chaturvedi, and Bharat B. Aggarwal. "Oxidative Stress, Inflammation, and Cancer: How Are They Linked?" Free Radical Biology and Medicine 49, no. 11 (2010): 1603-616. doi:10.1016/j.freeradbiomed.2010.09.006.

Seiden, M. V. "Ovarian Cancer." The Oncologist 6, no. 4 (2001): 327-32. doi:10.1634/theoncologist.6-4-327. 
Sun, Zhuoyu, Lucy Gilbert, Antonio Ciampi, Jay S. Kaufman, and Olga Basso. "Estimating the Prevalence of Ovarian Cancer Symptoms in Women Aged 50 Years or Older: Problems and Possibilities." American Journal of Epidemiology 184, no. 9 (November 13, 2016): 670-80. Accessed March 15, 2019. doi:10.1093/aje/kww086.Walker, M., M.D., \& Sobel, M., M.D. (2018). Diagnosing ovarian cancer. Canadian Medical Association.Journal, 190(42) doi:http://dx.doi.org.molloy.idm.oclc.org/10.1503/cmaj.180499

Wang, Ying-Jie, and Meenhard Herlyn. "The Emerging Roles of Oct4 in Tumor-initiating Cells." American Journal of Physiology-Cell Physiology 309, no. 11 (2015). doi:10.1152/ajpcell.00212.2015.

Zhang, Hui, Xiaofang Zhang, Xiaojuan Wu, Weiwei Li, Peng Su, Hongxia Cheng, Lei Xiang, Peng Gao, and Gengyin Zhou. "Interference of Frizzled 1 (FZD1) Reverses Multidrug Resistance in Breast Cancer Cells through the Wnt/B-catenin Pathway." Cancer Letters 323, no. 1 (2012): 106-13. doi:10.1016/j.canlet.2012.03.039.

Zhang, Xiang, Zhen Zhang, Qing Zhang, Quansheng Zhang, Peiqing Sun, Rong Xiang, Guosheng Ren, and Shuang Yang. "ZEB1 Confers Chemotherapeutic Resistance to Breast Cancer by Activating ATM." Cell Death \& Disease 9, no. 2 (2018). doi:10.1038/s41419-017-0087-3. 